\title{
Endoscopic removal and replacement of the same lumen-apposing metal stent for multiple endoscopic necrosectomy sessions $\square$
}

\author{
Authors \\ Rishabh Gulati, Tarun Rustagi \\ Institution \\ Division of Gastroenterology and Hepatology, University of \\ New Mexico, Albuquerque, New Mexico, United States \\ submitted 18.1.2020 \\ accepted after revision 2.6.2020 \\ published online 2.6.2020 \\ Bibliography \\ Endoscopy 2021; 53: 71-74 \\ DOI 10.1055/a-1192-3219 \\ ISSN 0013-726X \\ (c) 2020. Thieme. All rights reserved. \\ Georg Thieme Verlag KG, Rüdigerstraße 14, \\ 70469 Stuttgart, Germany \\ Corresponding author \\ Tarun Rustagi, MD, Division of Gastroenterology and \\ Hepatology, University of New Mexico School of Medicine, \\ 1 University of New Mexico, MSC10-5550, Albuquerque, NM \\ 87131, United States \\ Fax: +1-505-2726839 \\ tarunrustagi06@gmail.com
}

\section{ABSTRACT}

Background This study evaluated the feasibility, efficacy, and safety of removal and replacement of a lumen-apposing metal stent (LAMS) to facilitate direct endoscopic necrosectomy (DEN).

Methods Endoscopic ultrasound-guided LAMS placement for walled-off necrosis (WON) was performed. During subsequent endoscopy, the LAMS was removed, DEN was performed, and the same LAMS was redeployed across the transmural tract.

Results 140 LAMS were placed for drainage (43 pseudocysts, 97 WON) from 10/1/2016 to $11 / 15 / 2019$, and 40 patients (mean age 43.9 [SD 15.4] years; $70 \%$ males; mean WON size 10.9 [SD 4.9] $\times 8.7$ [SD 3.8] cm) underwent removal and replacement of the same LAMS for multiple DEN sessions. LAMS was successfully replaced across the transmural tract during all 81 DEN sessions. Complete resolution was achieved after a median of 2 DEN sessions (range 1-7; mean 2.4 [SD 1.5]), with stent retrieval after a mean of 64.1 (SD 31.6) days after initial placement. No stent damage, tissue ingrowth, or major bleeding occurred.

Conclusions Removal and replacement of LAMS during multiple necrosectomies is feasible, safe, and facilitates DEN.

\section{Introduction}

Direct endoscopic necrosectomy (DEN) is a minimally invasive technique that allows transmural access and removal of solid necrotic debris from a pancreatic walled-off necrosis (WON) [1-3]. Development of the "barbell-shaped" lumen-apposing metal stent (LAMS) has facilitated DEN with high clinical success and safety $[4,5]$. However, the amount of necrotic material extracted is limited by the inner diameter and flanges of the stent, with large WONs requiring multiple DEN sessions through the stent. We hypothesized that removal of the LAMS can facilitate DEN by improving endoscope maneuverability through the cystgastrostomy or cystenterostomy, thereby allowing greater access to WON and aid the removal of larger solid necrotic debris. The aim of this study was to evaluate the feasibility and safety of this novel modification that involves removal and replacement of the same LAMS to facilitate DEN.

\section{Methods}

A retrospective analysis of all patients undergoing endoscopic drainage of pancreatic fluid collections (PFC) at our institution between 1 October 2016 and 15 November 2019 was performed. Patients with WON who underwent endoscopic ultrasound (EUS)-guided drainage via cautery-enhanced LAMS (CELAMS; Boston Scientific, Marlborough, Massachusetts, USA) with more than one DEN session were included in the study. Linear-array echoendoscope (GF-UCT180; Olympus America, Center Valley, Pennsylvania, USA) was used for initial placement of the CE-LAMS. The regular upper endoscope (GIF-HQ190; Olympus America) was used for all removal and replacement of LAMS and for all DEN procedures.

All patients underwent fluoroless EUS-guided CE-LAMS placement using the direct free-hand technique for perigastric or periduodenal WON at the first endoscopic session. DEN was not performed during the initial session. During subsequent endoscopy, if residual solid necroses was noted at esophagogastroduodenoscopy and/or EUS, the decision was made to 

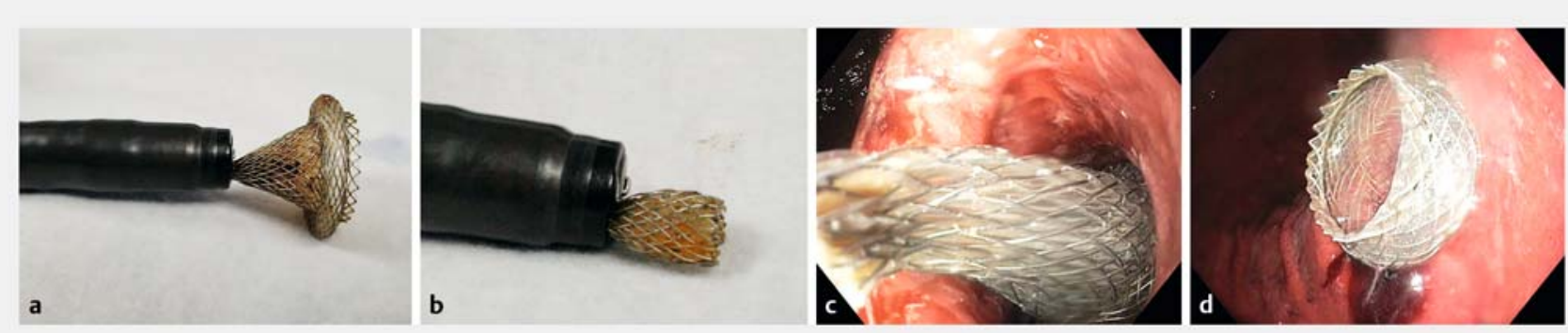

Fig. 1 Replacement of a lumen-apposing metal stent (LAMS) after direct endoscopic necrosectomy. a, b The LAMS was manually inserted inside the distal tip of the upper endoscope. $\mathbf{c}, \mathbf{d}$ The LAMS was redeployed across the cystgastrostomy at the end of each session.

proceed with DEN. The LAMS was then removed using a disposable snare (Spiral Snare; Olympus America) or a reusable Alligator Jaw grasping forceps (Olympus America) and placed within a clean container with sterile water. The WON cavity was then entered through the cystgastrostomy or cystenterostomy tract to perform DEN in the standard fashion using various devices including snares (Spiral Snare) and forceps (Raptor - US Endoscopy, Mentor, Ohio, USA; rat-toothed forceps - Olympus America) as previously described [6,7]. At the end of the DEN session, if significant residual solid necrotic material remained, the previously removed LAMS was placed back into position across the transmural tract. This was performed by first manually reinserting the LAMS inside the distal end of the working channel of the gastroscope. Once the scope was in position inside the cyst cavity, the distal flange of the LAMS was extruded by applying forward pressure using a forceps device inserted inside the working channel of the scope. The proximal flange was then deployed in the gastric or duodenal lumen, across the matured cystogastrostomy or cystduodenostomy tract ( $\triangleright$ Fig. 1, - Video 1). At each subsequent DEN session, this process was repeated until the WON cavity was emptied of solid debris. All patients were advised to discontinue acid suppression therapy to allow gastric acid to enter the WON cavity and aid the breakdown of necrotic material.

\section{Results}

A total of 216 patients underwent CE-LAMS placement at our institution from 1 October 2016 to 15 November 2019. Of these, 140 patients had PFCs and 76 had non-PFC indications for placement of LAMS ( $\mathbf{F i g . 2}$ ). A total of 97 patients (69\%) had WON that required endoscopic drainage. Of these, 40 patients who required more than one DEN session, and in whom the LAMS removal and replacement technique was utilized, were included in the study. The mean age was 43.9 years (standard deviation [SD] 15.4) and 70\% were male. Etiologies for acute necrotizing pancreatitis in these 40 patients included gallstones/biliary sludge $(n=18)$, alcohol $(n=10)$, hypertriglyceridemia $(n=3)$, drug-induced $(n=3)$, and idiopathic $(n=6)$. The mean size of WON was 10.9 [SD 4.9] $\times 8.7$ [SD 3.8] cm.

All patients had transgastric LAMS (cystgastrostomy), except for one patient who had cystduodenostomy with trans- duodenal LAMS placement. All but one of the stents used were of $15 \times 10 \mathrm{~mm}$ configuration; the other one was $20 \times 10 \mathrm{~mm}$. The mean interval from initial EUS-guided LAMS placement to next DEN session was 16 [SD 12.7] days. The mean interval between subsequent DEN sessions was 21.5 [SD 16.4] days. LAMS was successfully removed and replaced across the cystgastrostomy or cystduodenostomy tract after all 81 DEN sessions (100 $\%$ success rate) with a mean of 2 [SD 1.4] stent redeployments per patient (range 1-6). LAMS was removed and replaced $\geq 3$ times in 10 patients ( $25 \%$ ) without difficulty. There was no difficulty in reloading any stent, including 20-mm LAMS. Removal of large necrotic pieces with each pass through the cystgastrostomy or cystduodenostomy tract was achieved ( $\triangleright$ Fig.3). This was evidenced by the removal of several large necrotic pie-

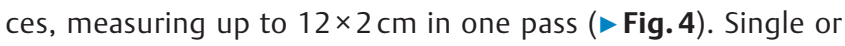
multiple 7-Fr double-pigtail plastic stents were placed across the LAMS in 24 patients (60\%).

There was no evidence of grossly visible stent damage or endoscopic evidence of tissue ingrowth within the stent. Complete resolution was achieved after a median of 2 DEN sessions

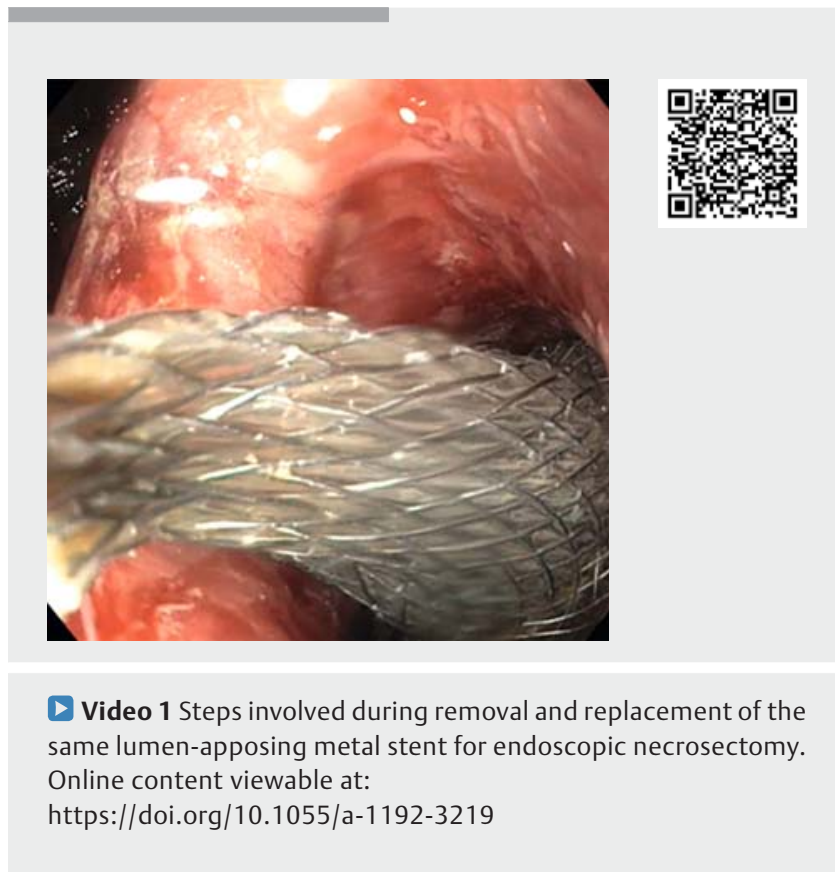




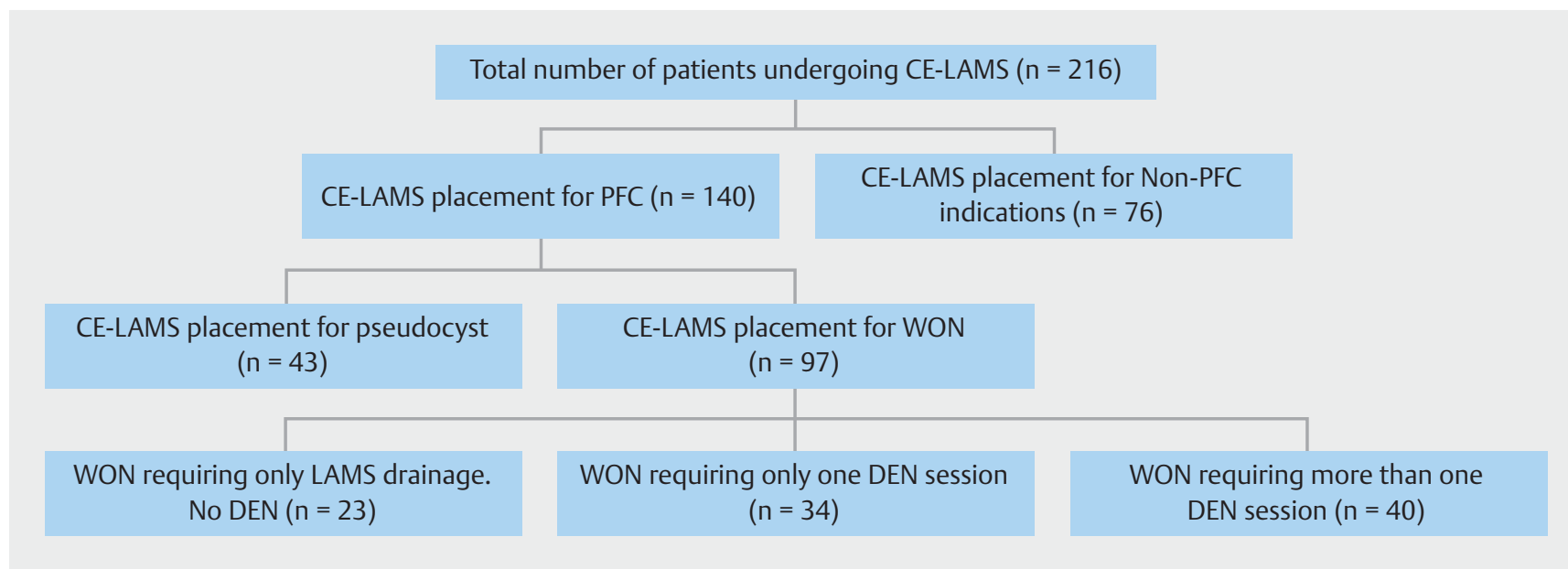

- Fig. 2 Flow diagram showing patients included in the study. CE-LAMS, cautery-enhanced lumen-apposing metal stent; PFC, pancreatic fluid collection; WON, walled-off necrosis; DEN, direct endoscopic necrosectomy.

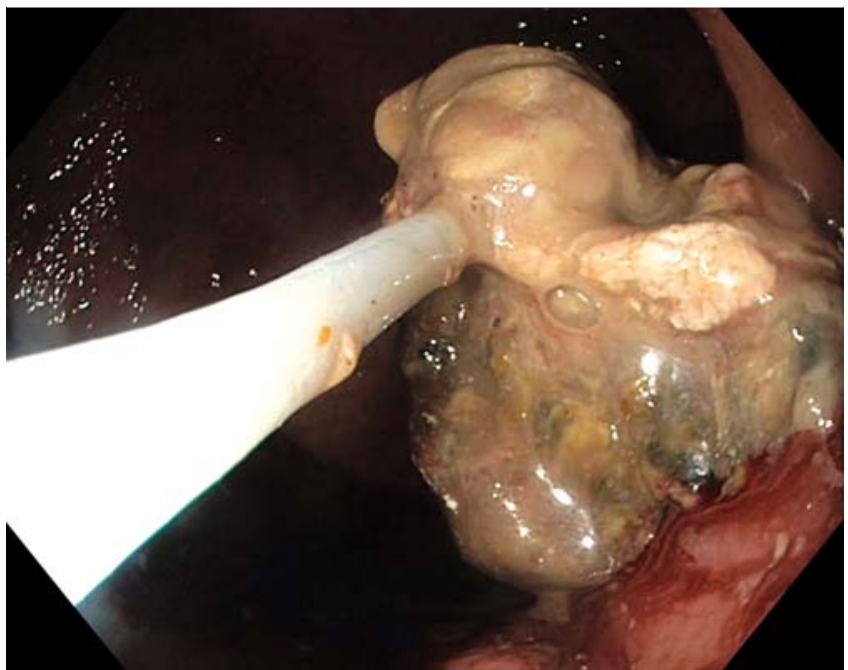

Fig. 3 Direct endoscopic necrosectomy using a snare, with removal of large necrotic pieces.

(range 1-7; mean 2.4 [SD 1.5]), with stent retrieval after a mean of 64.1 [SD 31.6] days after initial placement. One patient was lost to follow-up and LAMS was not removed. There was one instance of spontaneous dislodgment of the stent when it was noted to be in the gastric lumen during subsequent endoscopy. There were no major bleeding episodes or other adverse events noted during the median follow-up of 25.1 weeks (range 1-109).

\section{Discussion}

EUS-guided drainage of WON followed by DEN has revolutionized the management of WON and is preferred over surgical necrosectomy given its low morbidity and mortality $[1,8]$. With the advent of LAMS in 2013, passage of the endoscope through the lumen of the stent into the cavity for mechanical necrosectomy became a reality. A significant limitation of any form of

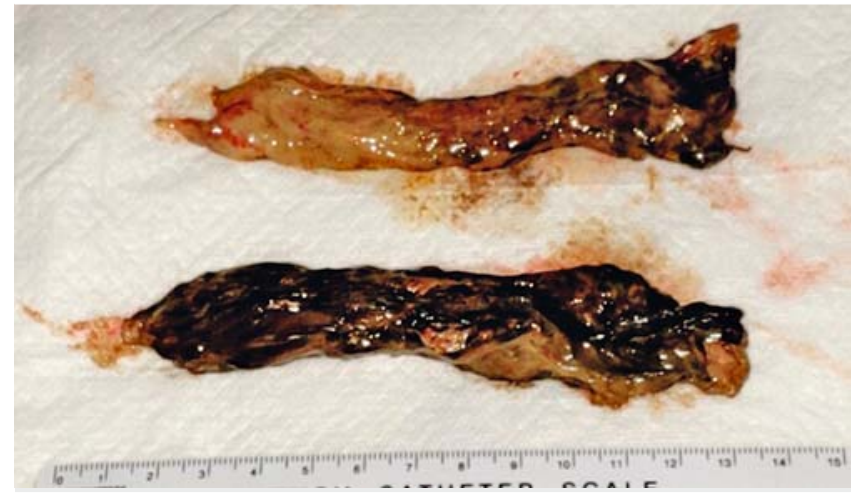

Fig. 4 Large solid necrotic pieces (measuring up to $12 \mathrm{~cm}$ ) were removed during necrosectomy using the lumen-apposing metal stent removal and replacement technique.

DEN is the time-consuming, tedious nature of the job. Even though the LAMS permits endoscopic entry into the cavity, it still requires the use of accessories that have not been designed to remove large amounts of necrotic tissue at a time. Given the current lack of dedicated endoscopic devices for DEN, several routinely available devices such as snares, grasping forceps, and nets have been used based on availability and endoscopist's experience and preference. This process can often be frustrating, leading to prolonged procedure times and multiple sessions.

One of the remarkable properties of LAMS is its elasticity and return to its barbell shape once the deforming force is removed. This allows it to be easily introduced into the distal end of the working channel of an adult upper endoscope and redeployed across the existing tract. Single-patient case reports of accidental stent dislodgment and successful replacement have been described recently while performing necrosectomy [9-11]. Based on these case reports and results from this study, we believe that this novel technique of LAMS removal and replacement would improve overall DEN procedure effi- 
ciency, reduction of endoscopist fatigue, and prove to be cost beneficial in cases of accidental migration or dislodgment.

Our study demonstrates that the removal and redeployment of the LAMS across the cystgastrostomy or cystenterostomy is technically feasible and safe in all cases. We believe this modification improves scope maneuverability including allowing for scope retroflexion within the WON cavity, which potentially improves visualization and debridement of deeper recesses of the necrotic cavity. In addition, removal of LAMS prior to DEN provides the potential benefit of removing large necrotic pieces intact with each pass, thus facilitating DEN with the perceived benefit of fewer passes and sessions with overall shorter procedure time needed. Indeed, fewer DEN sessions were required in the current study compared with the results of a recent metaanalysis (2.4 vs. 4.09; $P<0.001)$ [12]. Although we used a standard (diagnostic) channel upper endoscope for all our procedures, we believe insertion of the stent, particularly $20-\mathrm{mm}$ LAMS, into the distal tip of the scope would be much easier using a therapeutic channel upper endoscope.

There are several limitations to this study. It was a referralbased, single tertiary center, retrospective analysis. There were no comparative or control groups available. Further controlled studies comparing standard DEN performed through LAMS vs. DEN without LAMS (using this novel technique) are needed to provide data on any differences in number of DEN sessions required and procedure times. However, as with most studies involving PFC and/or WON, this is a difficult task. These cavities are heterogeneous with varying degrees of composition and consistency of necrotic material, making any comparative quantification difficult. Several other variables could also affect the outcomes, such as location and size of WON, use of dilute hydrogen peroxide for lavage, and endoscopist experience with DEN. In the future, use of larger-bore 20-mm LAMS for larger WON, and development and use of dedicated necrosectomy devices will likely aid in DEN and can further reduce the number of DEN sessions. Finally, given potential concerns from product manufacturers and ethical issues with the reuse of LAMS, we recommend using this technique under an institutional review board-approved protocol.

In summary, we describe a novel and safe technique of removal and replacement of the LAMS to facilitate DEN. Further controlled studies are needed to confirm the benefit of this technique in reducing procedure duration and number of DEN sessions.
Competing interests

The authors declare that they have no conflicts of interest.

\section{References}

[1] Baron TH, DiMaio C], Wang AY et al. American Gastroenterological Association Clinical Practice Update: Management of pancreatic necrosis. Gastroenterology 2020; 158: 67-75.e1

[2] Baron TH, Thaggard WG, Morgan DE et al. Endoscopic therapy for organized pancreatic necrosis. Gastroenterology 1996; 111: 755-764

[3] Seewald S, Groth S, Omar S et al. Aggressive endoscopic therapy for pancreatic necrosis and pancreatic abscess: a new safe and effective treatment algorithm (videos). Gastrointest Endosc 2005; 62: 92-100

[4] Itoi T, Binmoeller KF, Shah J et al. Clinical evaluation of a novel lumenapposing metal stent for endosonography-guided pancreatic pseudocyst and gallbladder drainage (with videos). Gastrointest Endosc 2012; 75: 870-876

[5] Shah RJ, Shah JN, Waxman I et al. Safety and efficacy of endoscopic ultrasound-guided drainage of pancreatic fluid collections with lumen-apposing covered self-expanding metal stents. Clin Gastroenterol Hepatol 2015; 13: 747-752

[6] Thompson CC, Kumar N, Slattery J et al. A standardized method for endoscopic necrosectomy improves complication and mortality rates. Pancreatology 2016; 16: 66-72

[7] Seifert H, Biermer M, Schmitt W et al. Transluminal endoscopic necrosectomy after acute pancreatitis: a multicentre study with longterm follow-up (the GEPARD Study). Gut 2009; 58: 1260-1266

[8] Bakker OJ, Van Santvoort HC, Van Brunschot S et al. Endoscopic transgastric vs surgical necrosectomy for infected necrotizing pancreatitis: a randomized trial. JAMA 2012; 307: 1053-1061

[9] Okuno M, Mukai T, Ichikawa $\mathrm{H}$ et al. Endoscopic replacement technique for migrated lumen-apposing metal stent during endoscopic pancreatic necrosectomy. VideoGIE 2019; 4: 220-222

[10] Mistry T, Shah M, Javia S et al. Retrieval and redeployment of migrated lumen-apposing metal stent to facilitate endoscopic necrosectomy of walled-off necrosis. VideoGIE 2018; 3: 151-152

[11] Guarner-Argente C, Colán-Hernández J, Concepción-Martín M et al. Replacement of the same lumen-apposing metallic stent for multiple necrosectomy sessions. Endoscopy 2015; 47: (Suppl. 01): E447-448

[12] Puli SR, Graumlich JF, Pamulaparthy SR et al. Endoscopic transmural necrosectomy for walled-off pancreatic necrosis: a systematic review and meta-analysis. Can J Gastroenterol Hepatol 2014; 28: 50-53 